Lanzetta, M.; Tantussi, G.: "Vision System Calibration and Sub-Pixel Measurement of Mechanical Parts"

AMST'99, Proceedings of the $5^{\text {th }}$ International Conference on

«Advanced Manufacturing Systems and Technology», CISM Courses and Lectures N. 406, ISBN:3-211-83148-7

Edited by E. Kuljanic, Springer-Verlag, Wien New York, Udine, Italy, June 3rd-4th, 1999, pp. 695-702

\title{
VISION SYSTEM CALIBRATION AND SUB-PIXEL MEASUREMENT OF MECHANICAL PARTS
}

\author{
M. Lanzetta \\ Department of Mechanical, Nuclear and \\ G. Tantussi \\ Production Engineering, University of Pisa
}

KEY WORDS: Measurement, Visual Inspection, Camera Calibration, Sub-pixel Algorithm

ABSTRACT: In this article a measuring system based on artificial vision techniques is described. Even if this problem is not new, several aspects, including sub-pixel algorithms, system programming and lighting, that are addressed in this paper, are still under study. Among them, the vision system calibration is focused. The theoretical model and the practical details of a fast method for 2-D problems are described, by comparing 3-D techniques already in the literature. The main features of the developed system are: cheap and easy to use. An example application is provided for the overall performance assessment.

\section{INTRODUCTION}

Even if many 3-D vision applications are available in the literature [1], most of industrial applications are still in 2-D. In metrology, non-contact techniques, including artificial vision, have a rapid diffusion both in the literature and in the industrial environment and have been widely approached; in particular, measuring machines based on optical techniques are commercially available. 
However, there are several open problems [2]: the main drawback of these methods is the low accuracy due to the optical distortion and to the low resolution of available sensors that requires the system calibration and the use of methods to increase the nominal spatial resolution, commonly addressed to as sub-pixel algorithms. In this paper we describe a simple, inexpensive and reliable vision system for metrology based on the integration of some of the more promising and fast techniques available in the literature.

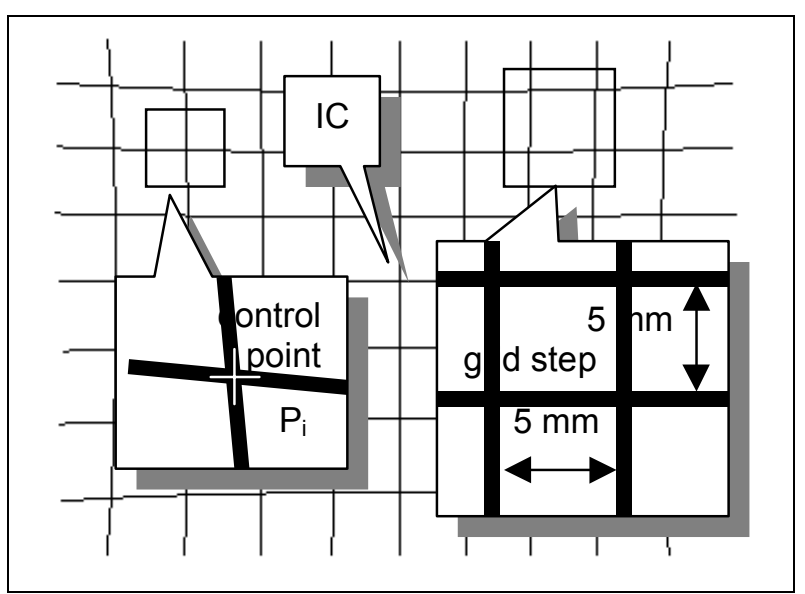

Figure 1 - Sample grid with cushion (negative) distortion

\section{CALIBRATION TECHNIQUES}

The vision system calibration is a key factor to achieve the maximum overall performance and is necessary in all cases when absolute measurement on images is performed [2,3]. It allows finding the correspondence between image points and workspace. The calibration program is executed ones after the system set-up, in order to determine the model parameters that are used every time an image is grabbed for part measurement purposes. The necessary parameters are $[1,3]$ : external, internal and optical distortion. The external parameters describe the camera position: translation $\mathrm{T}_{3 \times 3}$ and orientation $\mathrm{R}_{3 \times 3}$ in some world coordinate system. The internal parameters are: the Image Centre (IC), that is the intersection between the image plane and the optical axis, the focal length $\mathrm{f}$, to determine the perspective projection, the image or pixel aspect ratio $A R$ and the scale factor $s\left(s_{x}\right.$ or $s_{y}$ if $\left.A R \neq 1\right)$ $[\mathrm{mm} /$ pixels]. The optical distortion caused by the lenses can be reduced below the maximum acceptable error in two ways: with software methods, increasing the hardware requirements and the processing time, or with more expensive optical devices, for instance by the use of a telecentric objective [4]. The maximum acceptable error in the case of digital imaging is one pixel or the sub-pixel algorithm accuracy, if any (§ 3). Several authors have proposed different techniques that can be characterised as follows [3]: methods based (i) on known world points, (ii) on the geometric relationships within the image, like the vanishing point of parallel lines, (iii) or assuming special hypotheses on perspective or requiring a particular system configuration (stereo view, hand-eye, etc.), or (iv) based on motion, from an image sequence, like in robot or active vision. However developers of artificial vision applications usually do not have the skill, patience and interest in laborious calibration methods. Therefore camera calibration must be simple and as quick as possible. Considering that the examined problem is in $2-\mathrm{D}$, as in many industrial applications, in order to exploit all available information, the system calibration is performed on a plane approximately perpendicular to the camera axis using a known pattern. Grids or spheres are common to many techniques, like [5] and [6]. The 2-D applications of these 3-D ap- 
proaches are compared and discussed here in all the calibration steps in $\S 2.1$ and 2.2, providing the parameters obtained for the used system. Concerning the external parameters, the translation and rotation matrices are represented, in the particular configuration used, by an identity matrix $\mathrm{R} \equiv$ $\mathrm{I}_{3 \times 3}$ and by the vector $\mathrm{T} \equiv(0$, $0, \mathrm{~T}_{\mathrm{z}}$ ). For the internal parameters and the optical distortion, the following methods have been used.

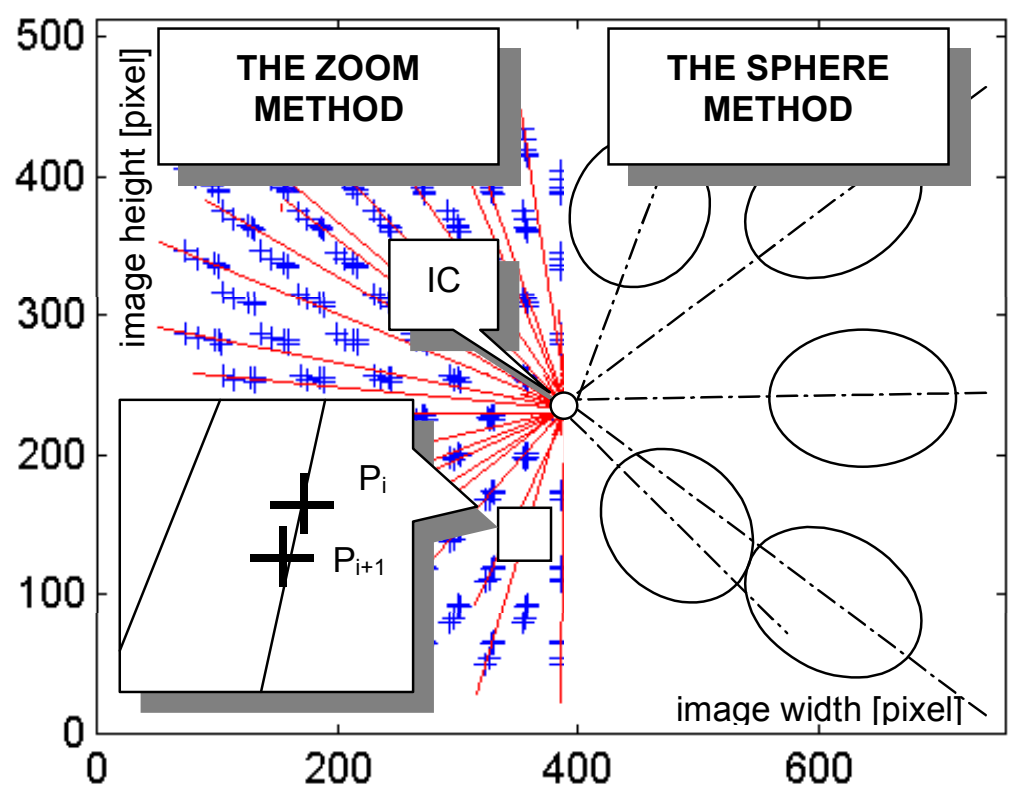

\subsection{The grid method}

Figure 2 - The grid and the sphere methods to find the Image Centre (IC)

This method is the 2-D application of [5]. A sample binary grid (Figure 1 and 2) is used whose intersection points represent the control points of this method. The IC is calculated first as it is weakly influenced by the more relevant optical distortion component, which is radial. Even if in 2-D problems a very accurate location of the IC is not necessary, it can be automatically found with a small effort. We assume that its position is zoom-independent. Consequently a zoom change produces a radial displacement of all image points using the IC as the pole. By changing the zoom, a general point $\mathrm{P}_{\mathrm{i}}$ moves to position $\mathrm{P}_{\mathrm{i}+1}$, and so forth (left detail of Figure 2). The straight lines displayed in Figure 2 (left) are determined with the least-squares method. A convergence study gave as a result that 5 positions of 60 control points provide the necessary and maximum accuracy.

The IC is calculated by minimising its distance function $\Delta$ from all the straight lines, whose coefficients in the implicit equation are $\mathrm{p}_{\mathrm{i}}, \mathrm{q}_{\mathrm{i}}$ and $\mathrm{r}_{\mathrm{i}}$ :

$$
\Delta=\sqrt{\frac{1}{N} \sum_{\mathrm{i}=1}^{\mathrm{N}} \mathrm{d}_{\mathrm{i}}^{2}} \quad \text { where } \quad \mathrm{d}_{\mathrm{i}}=\frac{\left|\mathrm{p}_{\mathrm{i}} \mathrm{Y}_{\mathrm{IC}}+\mathrm{q}_{\mathrm{i}} \mathrm{X}_{\mathrm{IC}}+\mathrm{r}_{\mathrm{i}}\right|}{\sqrt{\mathrm{p}_{\mathrm{i}}^{2}+\mathrm{q}_{\mathrm{i}}^{2}}}
$$

Equation (1) is numerically solved to find the IC co-ordinates $\left(X_{I C}, Y_{I C}\right) \equiv(230,390)$. After setting the zoom in order to maximise the field of view with respect to the observed object, the magnification factor is assessed as the ratio $T_{z} / f=15.337$, using the control points belonging to the less distorted image area (column 1 of Table I). By evaluating this ratio instead of the two values separately, the singularity coming from the 2-D application of the grid method is solved.

The aspect ratio $\mathrm{AR}$, has been determined as the average ratio between the square sides of 
the grid on all the images grabbed to find the IC; $\mathrm{AR}=0.9514$ has been determined considering the non-distorted (central) control points. To find the optical distortion, the tangential distortion is neglected and to assess the radial distortion, regression is performed between the measured and the actual Euclidean distance of control points from the IC as in [7]. A $2^{\text {nd }}$ order interpolation equation is found, dividing the image in four concentric areas (Figure 3).

\subsection{The sphere method}

This method [6] is based on geometrical and optical considerations. The projection on the image plane of a sphere whose centre does not belong to the optical axis is an ellipse whose main axis is radial and contains the IC (Figure 2, right). A rear illuminated black sphere with known radius and tolerance has been used. The principal axes of ellipses are determined with the least-squares method and for the IC again equation (1) is used: $\mathrm{IC} \equiv(235$, 399). Geometric relations between the focal length and the ellipse features (eccentricity, principal axis, and boundary position) can be expressed [6]. With this method, the aspect ratio $\mathrm{AR}$ is given by the ratio between the two axes of each ellipse.

The sphere method for 2-D problems has generally a lower accuracy than the grid method as a consequence of several practical disadvantages:

- black spheres with low tolerances

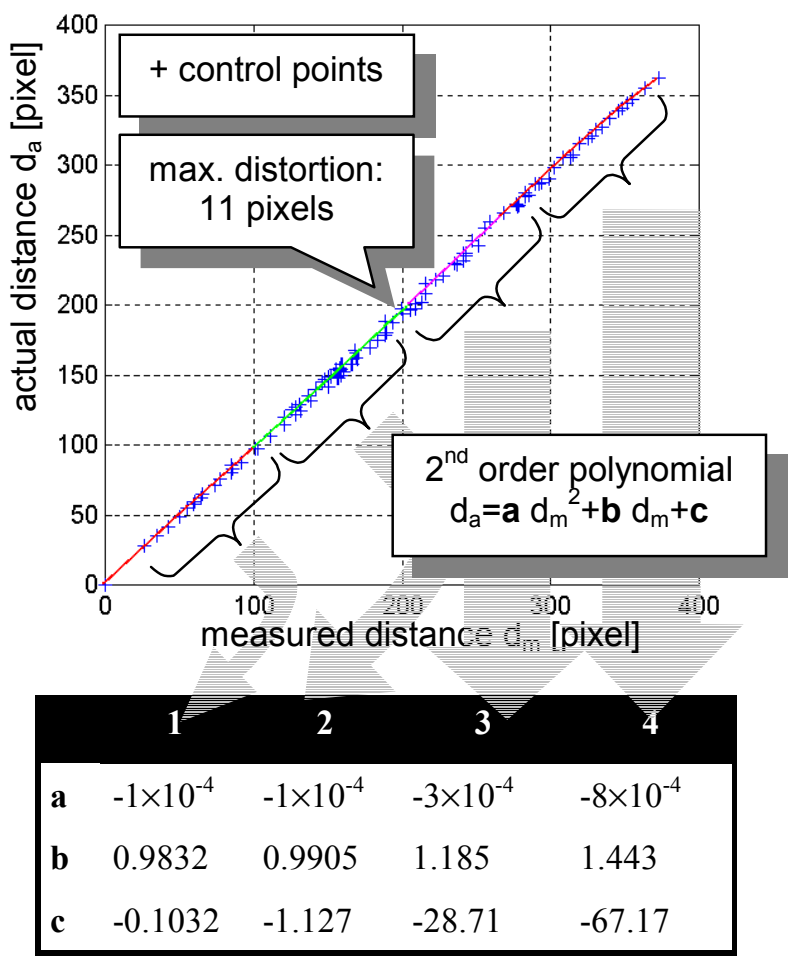

Figure 3 (above) - Interpolation of control points

Table I (below) - Polynomial coefficients in the four concentric areas are more difficult to find than laser printing a black grid. However a sharper grid (Figure 1 and 2) has been obtained with a plotter, a $0.1 \mathrm{~mm}$ pen and a transparent sheet for rear illumination;

- the sample positioning and lighting is also more critical;

- focusing a sphere requires a higher field depth than a 2-D object;

- the mathematical computation of the ellipse implicit equation is heavier than the grid points regression and errors on parameters are higher;

- the axis intersection region (near the IC in Figure 2) is larger with this method, because of image processing errors (edge detection with out-of-focus); however the error compensation due to the problem symmetry reduces the impact of this error source. 
The direct consequence of the experimental comparison is that the 2-D application of the grid method is easier and yields a higher accuracy than the sphere method and for these reasons it has been integrated in the developed system.

\subsection{The optical distortion symmetry} method

The basic idea of this new method to find the IC is the sample image symmetry: the lens should produce a symmetrical distortion with respect to the two image axes.

a.

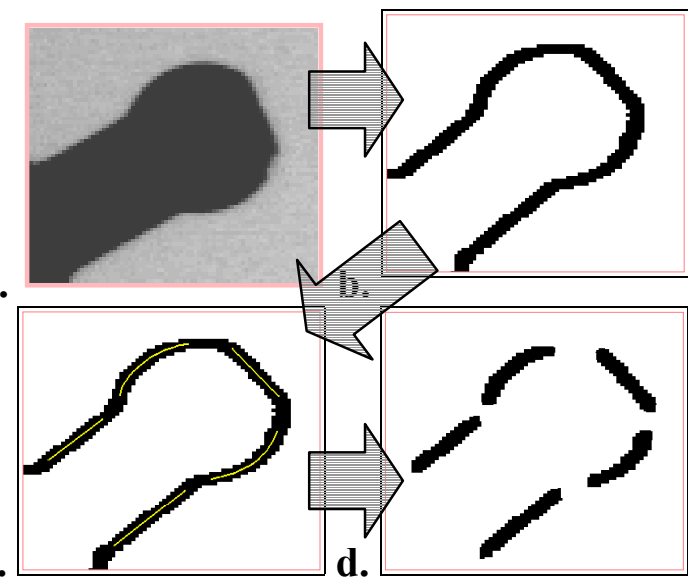

Figure 4 - The main steps of the algorithm The four functions representing the actual distance of control points located in the four quadrants $d_{a}$ versus the measured distance $d_{m}$, using the correct IC, are symmetrical two by two. For low cost lenses, the radial symmetry centre could be not coincident with the IC, but for the above reasons it represents a good estimation of it. This method is more suitable for wide-angle lenses, but it cannot be applied to the used system, as the optical distortion is lower than the convergence step and the algorithm becomes unstable, because the error estimating the branch symmetry calculating the polynomial of Figure 3 is of the same order of the iterative accuracy increase at each step. A geometrical interpretation is that, with our system, the four branches match in an area that is greater than the error to estimate.

\section{SUB-PIXEL MEASUREMENT}

The nominal spatial resolution $\mathrm{R}_{\mathrm{N}}$ is the ratio between the field of view and the sensor resolution. For a higher accuracy and consequently a higher measurement reliability, a better (lower) resolution $\mathrm{R}_{\mathrm{N}}$ is necessary that can be obtained with the following methods:

- by increasing the sensor resolution;

- by reducing the field of view, divided in smaller areas, with a relative movement between camera and object, like in [2];

- with a sub-pixel method, such as the one described, or with multiple acquisitions. Assuming a Gaussian distribution of errors, when using $\mathrm{N}_{\mathrm{p}}$ points, the position accuracy is increased and $\mathrm{R}_{\mathrm{N}}$ is reduced by $\alpha=\sqrt{\mathrm{N}_{\mathrm{P}}}$.

More details concerning the impact of the edge width on resolution can be found in [2]. 


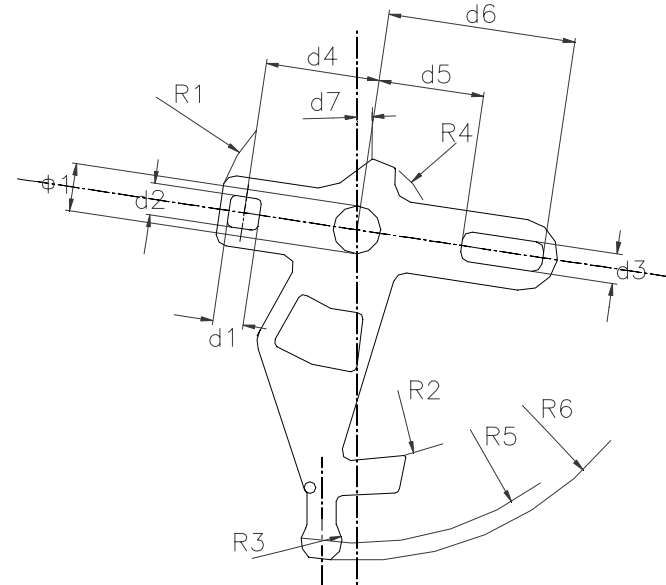

Figure 5 - Benchmark: «blocking lever» (dimensions in Table II)
This method can be applied (i) to flat object or (ii) when the boundaries belong to one or more planes perpendicular to the optical axis, if their edges can be represented with geometric primitives. The main steps of the algorithm are summarised in Figure 4:

a. image acquisition with rear illumination [8];

b. edge-detection, obtained with a binarisation and a convolution;

c. optical correction and segmentation;

d. least-squares interpolation of primitives [9].

A binarisation threshold is easy to find on a bimodal histogram (Figure 4a.). However a lighting or threshold change may affect

the edge width and position because of light scattering effects. This problem is solved by using a reference dimension on the observed part and by finding the binarisation threshold with an exhaustive method, until the correct dimension is achieved.

After edge-detection, data are compressed and the boundary co-ordinates only are kept instead of the whole image, as calibration involves those data only. The necessary memory is reduced, in the described example, from an 8 bit (256 grey-level) $756 \times 567$ image, to $n_{p}$ couples of 10 bit co-ordinates. The interpolation function depends on the geometric primitive, whose main parameters are obtained using the implicit equations, e.g. a straight line or a circle for a segment or an arc respectively.

\section{THE LEARNING PROGRAM}

The inspection planning is manually performed by self-learning as in most commercial packages. The operator provides the system with the critical dimensions and their position by splitting the contour of the grabbed image into geometrical primitives with a graphical interface and a pointing device according to a decision tree. Every primitive is selected through a set of points to define a window depending on the primitive being searched and according to optimal search criteria. Regarding the system engineering, programming can be automated using CAD data as described in [9]. The primitive selection (segmentation) could also be automated finding the knot-points between two primitives with the method in [10]. The system is able to measure a part in any position within the field of view with a pattern matching technique, like in [3], therefore it can be used directly on-line without special fixtures. A template of the part to be measured is stored in memory in order to find the actual displacement and the angle of rotation with respect to the template. The present part position and orientation information can be used to shift and rotate the search mask containing the user-programmed windows. This method is preferable with respect to 
rotating the image, because the accuracy reduction due to pixel calculation (up to \pm 0.5 pixel) may affect the measurement, while the same error is acceptable when rotating the search mask.

\section{AN EXAMPLE APPLICATION AND THE EX- PERIMENTAL SET-UP}

The described system has been designed to be exploited for mechanical part measurement in a transfer pressing line for $100 \%$ part and process control purposes. At present a statistical control is performed (i) with a go-nogo calliper by an operator, and (ii) with a CMM for the remaining critical dimensions. If an error is detected, all parts manufactured and stored in a buffer since the previous control are rejected. As an example, a sheared part (Figure 5) has been used to test the system, made of the following components: a PC with a Matrox Image frame grabber, and a monochrome CCD matrix camera with a resolution of $756 \times 567$ pixels at 256 grey levels, with zoom lens. The best lighting performance has been obtained with a rear illumination, using a Fresnel lens instead of a

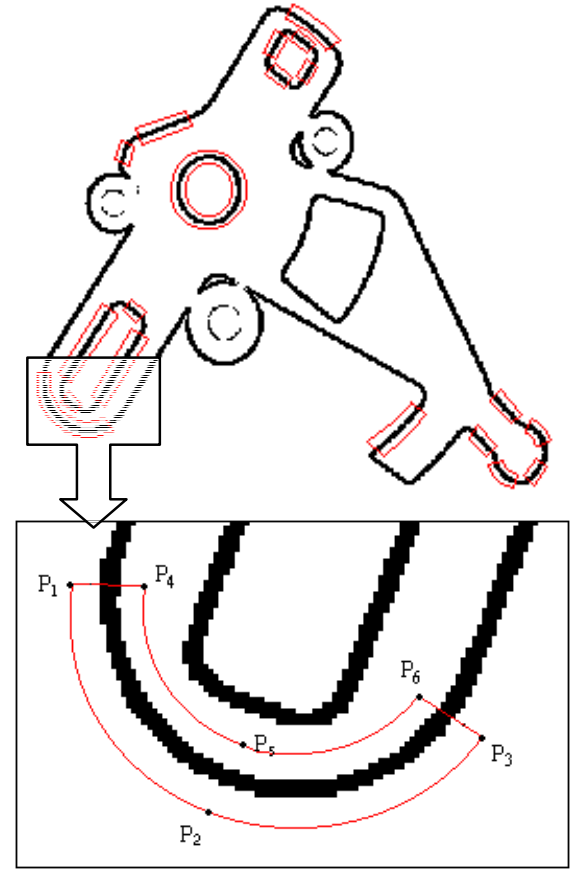

Figure 6 - The inspection mask. Detail of the search window for an arc, defined by six points simple surface diffuser. Such collimated light source with parallel rays has the following advantages: (i) it avoids reflections on the vertical surfaces for thick sheet parts; (ii) for 3-D objects, where edges are not in the same plane, it reduces the disturbances due to shadows and to the curved surface reflections. Using a fixed threshold binarisation, the light distribution should be as uniform as possible. This can be obtained with a proper lamp positioning or by software means, using a solid sample to find the correction coefficients. The effect of the external environmental lighting can be reduced increasing the rear illumination intensity and reducing the exposure time with a shutter, in order to keep a high signal-to-noise ratio. Tests have shown that a change of light intensity produces a measurement error of about $0.02 \mathrm{~mm}$ on $\phi 1$ (Table II) for a grey-level threshold change from 50 to 110 .

\section{RESULTS}

In Table II, the nominal dimensions $\mathrm{D}$ and the tolerances $\mathrm{t}$ of the observed part are compared with those coming from the vision system $\mathrm{D}_{\mathrm{m}}$ and those taken with standard workshop methods $D_{a}$. In the examined case, with a field of $100 \times 75 \mathrm{~mm}, R_{N}=0.13[\mathrm{~mm} /$ pixel] and it is not sufficient with the requirements in Table II. For this reason, the sub-pixel algorithm implemented was necessary. The low system resolution is enhanced in detail of Fig- 
Table II - The vision system performance

\begin{tabular}{|c|c|c|c|c|c|c|}
\hline Ref. & $\begin{array}{c}\mathbf{D} \\
{[\mathbf{m m}]}\end{array}$ & $\begin{array}{c}\mathbf{t} \\
{[\mathbf{m m}]}\end{array}$ & $\begin{array}{c}\mathbf{D}_{\mathbf{m}} \\
{[\mathbf{m m}]}\end{array}$ & $\begin{array}{c}\mathbf{D}_{\mathbf{a}} \\
{[\mathbf{m m}]}\end{array}$ & $\begin{array}{c}\mathbf{E} \\
{[\mathbf{m m}]}\end{array}$ & $\begin{array}{c}\text { \% } \\
\text { error }\end{array}$ \\
\hline$\phi_{1}$ & 8.1 & $\left(\begin{array}{c}+0.1 \\
0\end{array}\right)$ & 8.14 & 8.15 & -0.01 & -0.1 \\
\hline $\mathrm{d}_{1}$ & 5.2 & $\left(\begin{array}{c}+0.1 \\
0\end{array}\right)$ & 5.26 & 5.29 & -0.03 & -0.6 \\
\hline $\mathrm{d}_{2}$ & 5.4 & $\left(\begin{array}{c}+0.1 \\
0\end{array}\right)$ & 5.46 & 5.42 & 0.04 & 0.7 \\
\hline $\mathrm{d}_{3}$ & 5.1 & $\left(\begin{array}{c}+0.1 \\
0\end{array}\right)$ & 5.16 & 5.14 & 0.02 & 0.4 \\
\hline $\mathrm{d}_{4}$ & 19.4 & $\left(\begin{array}{c}+0.1 \\
-0.1\end{array}\right)$ & 19.36 & 19.34 & 0.02 & 0.1 \\
\hline $\mathrm{d}_{5}$ & 18 & $\left(\begin{array}{c}0 \\
-0.2\end{array}\right)$ & 17.92 & 17.90 & 0.02 & 0.1 \\
\hline $\mathrm{d}_{6}$ & 32 & $\left(\begin{array}{c}+0.2 \\
0\end{array}\right)$ & 32.02 & 30.06 & -0.04 & -0.1 \\
\hline $\mathrm{d}_{7}$ & 2.7 & $\left(\begin{array}{c}+0.1 \\
0\end{array}\right)$ & 2.80 & 2.77 & 0.03 & 1.1 \\
\hline $\mathrm{R}_{1}$ & 24 & $\left(\begin{array}{c}+0.1 \\
-0.1\end{array}\right)$ & 24.02 & 24.05 & -0.03 & -0.1 \\
\hline $\mathrm{R}_{2}$ & 39 & $\left(\begin{array}{c}+0.1 \\
-0.1\end{array}\right)$ & $38.80^{*}$ & 38.92 & -0.12 & -0.3 \\
\hline $\mathrm{R}_{3}$ & 3.5 & $\left(\begin{array}{c}0 \\
-0.1\end{array}\right)$ & 3.48 & 3.49 & -0.01 & -0.3 \\
\hline $\mathrm{R}_{4}$ & 12.3 & $\left(\begin{array}{c}+0.1 \\
0\end{array}\right)$ & $12.26^{*}$ & 12.34 & -0.08 & -0.7 \\
\hline $\mathrm{R}_{5}$ & 53 & $\left(\begin{array}{c}+0.1 \\
-0.1\end{array}\right)$ & $52.84 *$ & 52.94 & -0.1 & -0.2 \\
\hline $\mathrm{R}_{6}$ & 56 & $\left(\begin{array}{c}0 \\
-0.2\end{array}\right)$ & 55.86 & 55.90 & -0.04 & -0.1 \\
\hline
\end{tabular}

ure 6. Some dimensions* in Table II depend on the position of a single point and not of a primitive. Their estimation could be easily improved by locating points as the intersection of primitives. Apart from them, the maximum absolute error of the vision system is $\mathrm{E}_{\max }=$ $0.04 \mathrm{~mm}$.

The calibration techniques used have reduced the optical distortion (Figure 3) from a maximum of $1.43 \mathrm{~mm}$ to acceptable values, as shown by the values of $\mathrm{E}$.

The system repeatability, calculated as the maximum error E measuring ten times the circle $\phi_{1}$ is $E_{\text {rep }}=0.02$.

The system resolution, expressed as the minimum detectable dimension change, has been assessed measuring parts with different values of $\phi_{1}$ and is $E_{\text {res }}=0.02$. The angular accuracy is a direct consequence of linear accuracy. In all cases $\mathrm{E}<<\mathrm{t}$, then the described optical measuring method can be applied for part inspection without sorting errors.

\section{CONCLUSIONS}

The main features of the described system are: cheap, fast and reliable. Low cost, required for industrial applications, is obtained by software means, which is an inexpensive method 
to increase the performance of commercial hardware in addition to adopting higher resolution cameras. The cycle-time (10 s) is not far from the production-rate $(4 \mathrm{~s})$ and could be easily reduced by software optimisation or with special purpose hardware. The system reliability has been assessed by direct comparison on measured parts. A sub-pixel algorithm, including optimal hardware calibration to stress the overall performance of standard cameras and lenses has been implemented. The described system, obtained by comparing the performance of available techniques from the literature, has attained the goal of 0.3 pixel resolution. A new technique, which could not be tested with the used system, has also been proposed.

\section{ACKNOWLEDGEMENTS}

The authors wish to thank Ing. Carlo Scarabeo for his contribution to this work and Ing. R. Sbrana and Mr. P. Gasperini from Atoma Roltra S.p.A. - Pisa (Magna Group) for providing the material and their technical support.

\section{REFERENCES}

[1] Lanzetta, M.: 3-D Vision in Production Processes, Part I - State of the Art (in Italian), Automazione e Strumentazione, n. 2, Year XLVI (February 1998), 155-164.

[2] Lanzetta, M.: Rasterisation of 2-D profiles with Unlimited Resolution through Artificial Vision Techniques: an Application to the Wood Industry, Proceedings of the $10^{\text {th }}$ International ADM Conference, Florence Italy, September $17^{\text {th }}-19^{\text {th }}(1997), 311-316$.

[3] Lanzetta, M.: Stereo Vision with Neural Networks (in Italian), Automazione e Strumentazione, n. 7, Year XLIV (July/August 1996), 107-116.

[4] N.N.: 55 Telecentric, Accurate Machine Vision, brochure of Computar, Japan.

[5] Li, M.; Lavest, J.-M.: Some Aspects of Zoom-Lens Camera Calibration, Report from CVAP, KTH Stockholm Sweden, TRITA-NA-9503, CVAP172 (February 1995).

[6] Stein, G.P.: Internal Camera Calibration using Rotation and Geometric Shapes, M.Sc. Thesis, MIT Boston Massachusetts (February 1993).

[7] Lonardo, P.; Bruzzone, A.: Application of image analysis to mechanical metrology, Proceedings of III AITEM Conf., Fisciano (SA) Italy, September $17^{\text {th }}-19^{\text {th }}$ (1997), 459-466.

[8] Schroeder, H.E.: Practical Illumination Concept and Technique for Machine Vision Applications, in Robot Sensors, vol. 1 - Vision, Springer Verlag (1986), 229-244.

[9] Chen, J.-M.; Ventura, J.A.: Shape analysis of complex profiles, Int. J. Mach. Tools Manufact., vol. 35, n. 3 (1994), 399-429.

[10]Wu, Q.M.; Rodd, M.G.: Boundary segmentation and parameter estimation for industrial inspection, IEE Proceedings-E, vol. 137, n. 4 (July 1990), 319-327. 